\title{
(6) OPEN ACCESS \\ Assessment of pulmonary function in amyotrophic lateral sclerosis: when can polygraphy help evaluate the need for non-invasive ventilation?
}

\author{
Tino Prell, Thomas M Ringer, Kara Wullenkord, Philipp Garrison, Anne Gunkel, \\ Beatrice Stubendorff, Otto W Witte, Julian Grosskreutz
}

- Additional material is published online only. To view please visit the journal online (http://dx.doi.org/10.1136/ jnnp-2015-312185)

Hans-Berger Department of Neurology, University Hospital Jena, Jena, Germany

Correspondence to Dr Tino Prell, Klinik für Neurologie, Universitätsklinikum Jena, Erlanger Allee 101, Jena 07747, Germany; tino.prell@med.uni-jena.de

Received 3 September 2015 Revised 9 December 2015 Accepted 18 December 2015 Published Online First 24 March 2016

\section{ABSTRACT}

Background Non-invasive positive-pressure ventilation (NPPV) is an established, effective, long-term treatment for patients with amyotrophic lateral sclerosis (ALS), but the correct indicators for the establishment of NPPV have not been defined.

Methods In this retrospective study, records (spirometry, nocturnal polygraphy, nocturnal blood gases) of 131 patients with ALS were reviewed in order to evaluate the role of polygraphy for prediction of respiratory failure in ALS.

Results The patient group reporting with versus without dyspnoea had significantly lower values on the revised ALS-Functional Rating Scale (ALSFRS-R), vital capacity (VC), forced VC (FVC), arterial oxygen saturation and arterial oxygen tension readings, including a higher apnoea-hypopnoea index. 23 patients, who did not report about dyspnoea, had an FVC of $<75 \%$. Nocturnal hypoventilation was observed in $67 \%$ of the patients with ALS independent of their ALSFRS-R. The patient group with nocturnal hypoventilation was characterised by a significantly lower VC, FVC and maximal static inspiratory pressure compared with the group without nocturnal hypoventilation. However, also in the absence of nocturnal hypoventilation, 8 patients had a VC $<50 \%$ as predicted.

Discussion Our study shows that in patients not reporting dyspnoea and having an FVC of $>75 \%$, nocturnal hypoventilation was observed in nearly every second patient. Therefore, for the question of whether NPPV should be initiated, polygraphy does not provide useful additional information if the FVC is already $<75 \%$ as predicted. However, in patients with more or less normal lung function parameters or where lung spirometry cannot perform adequately (eg, bulbar ALS), it can provide sufficient evidence for the need of NPPV.

\section{INTRODUCTION}

Amyotrophic lateral sclerosis (ALS) is a fatal neurodegenerative disease characterised by the loss of upper and lower motor neurons, causing progressive muscular weakness of the limbs (limb onset) or dysarthria and dysphagia (bulbar onset). The average life expectancy is 3 years from the onset of symptoms, and patients usually die due to respiratory failure. Respiratory function is an important predictor of survival and quality of life for patients with ALS. During disease course, all three muscular components of the respiratory system (inspiratory, expiratory and bulbar muscles) decline, leading to a progressive decline in vital capacity (VC) and an increase in the work of breathing. Rapid shallow breathing, the inability to take deep breaths and the retention of mucus causes microatelectasis and decreased lung and chest wall compliance. Neuromuscular disorders may also generally be associated with obstructive or central sleepdisordered breathing, particularly during rapid eye movement sleep. ${ }^{1}$

Non-invasive positive-pressure ventilation (NPPV) is an established, effective, long-term treatment for patients with ALS that uses a face or nasal mask and a volume cycled or bi-level pressurelimited ventilator to provide an intermittent positive pressure to support ventilation. There is clear evidence that NPPV may be associated with improvement of sleep-related symptoms and quality of life. In addition, it is possible that early treatment using NPPV may offer a survival benefit, particularly in the subgroup of participants with normal or moderately impaired bulbar function. ${ }^{2-4}$ The median time from ALS diagnosis to death was significantly longer when NPPV was initiated earlier in the disease course, that is, when forced VC (FVC) was $>65 \%$ as predicted. ${ }^{5}$ However, questions concerning NPPV remain: What are the correct indicators for the establishment of NPPV in patients with ALS and at what time should it begin?

There are several measures of respiratory muscle strength. A FVC $<50 \%$, seated or supine, has been widely applied as the threshold to initiate NPPV. Moreover, measurements of respiratory muscle strength, nocturnal gas exchange and symptomatic complaints are used as indicators to start NPPV. Several consensus statements also recommend starting NPPV when daytime hypercapnia and nocturnal hypoventilation are present, when maximum inspiratory pressure is $<60 \mathrm{~cm} \mathrm{H}_{2} \mathrm{O}$, or if nocturnal arterial oxygen saturation $\left(\mathrm{artSpO}_{2}\right)$ is $<88 \%$ for more than five consecutive minutes. ${ }^{7}$ However, these recommendations are not based on controlled studies and none were developed selectively for ALS. Detecting early respiratory dysfunction in ALS is important, but is proving to be problematical. Needle electromyography (EMG) seems to be sensitive measure of early respiratory failure in ALS, but it is usually not a routine component. ${ }^{8} 9$ Therefore, in daily routine, one has to use several measures of respiratory muscle strength to monitor the progression of respiratory muscle weakness in 
ALS, because there is no single respiratory test available which can be used to reliably predict the onset of respiratory failure. We wanted to study the usefulness of polygraphy to predict respiratory failure. In contrast to polysomnography, the polygraphy represents an alternative, less time-consuming and costconsuming method measuring sleep parameters without utilising EEG recordings. Although analysis of sleep architecture (sleep states) is impossible, detailed information of nightly respiration, together with peripheral blood oxygenation, are provided. In this study, we performed a systematic evaluation of all consecutive polygraphies of our patients with ALS within 5 years compared with the corresponding detailed spirometry and clinical data. Thereby, this study should determine in which cases polygraphy supplies additional information for the necessity of NPPV in patients with ALS.

\section{MATERIALS AND METHODS}

\section{Participants and records}

Records were reviewed of all patients with ALS who were referred to our Department of Neurology between 02/2009 and 08/2014 (for epidemiological data, see table 1). Those patients who fulfilled El Escorial criteria for 'definite', 'probable' or 'laboratory supported' ALS and in whom spirometry, polygraphy and clinical data were available at the same time point ( \pm 3 days) were included in this study, resulting in a total of 131 patients with ALS. Patients with a family history of ALS were found to carry several mutations (1 with C9orf72 hexanucleotide repeat; 4 with SOD1 mutation), but all others reported no family history of ALS or frontotemporal dementia. However, since not all patients were genetically tested, we cannot rule out that more patients in our cohort have the C9orf72 mutation. None of the patients had thyroid dysfunction. The spirometry included VC (\% predicted), FVC (\% predicted) and maximal static inspiratory pressure (Pimax). For respiratory polygraphy,

Table 1 Clinical data of patients with ALS and onset subtypes

\begin{tabular}{lllll}
\hline & All & $\begin{array}{l}\text { Bulbar } \\
\text { onset }\end{array}$ & $\begin{array}{l}\text { Limb } \\
\text { onset }\end{array}$ & p Value* \\
\hline $\begin{array}{l}\text { Patients with ALS, } \mathrm{n} \\
\text { Male/female ratio }\end{array}$ & 131 & 46 & 85 & \\
$\begin{array}{l}\text { Age, years } \\
\quad \text { Mean }\end{array}$ & $75: 56$ & $22: 24$ & $53: 32$ & NS \\
$\quad$ SD & 61.0 & 65.3 & 58.5 & 0.009 \\
BMI & 12.4 & 10.7 & 12.6 & \\
$\quad$ Mean & & & & \\
$\quad$ SD & 23.9 & 23.7 & 24.0 & NS \\
ALSFRS-R & 3.6 & 3.5 & 3.6 & \\
$\quad \begin{array}{l}\text { Mean } \\
\text { SD }\end{array}$ & 32.21 & 31.78 & 32.46 & NS \\
$\begin{array}{l}\text { Subscore 4 ALSFRS-R } \\
\quad \text { Mean }\end{array}$ & 8.50 & 9.50 & 7.93 & \\
$\quad$ SD & 10.31 & 10.04 & 10.46 & NS \\
Disease duration, months & 2.00 & 1.98 & 2.02 & \\
$\quad \begin{array}{l}\text { Mean } \\
\text { SD }\end{array}$ & 20.55 & 20.22 & 20.73 & NS \\
$\begin{array}{l}\text { Prevalence of dyspnoea, } \mathrm{n} \\
\text { Prevalence of sleep problems, } \mathrm{n}\end{array}$ & 12.89 & 12.70 & 12.91 & \\
\hline
\end{tabular}

${ }^{*} p$ Values show differences between bulbar and limb onset by using $t$ test. ALS, amyotrophic lateral sclerosis; ALSFRS-R, ALS-Functional Rating Scale; BMI, body mass index; NS, non-significant. we used the Somnoscreen from SOMNOmedics (Randersacker, Germany), which recorded pulse oximetry, oronasal pressure and flow using a thermistor, chest/abdomen movements by impedance plethysmography, body position by a position sensor, snoring by microphone and heart rate and $\mathrm{SpO}_{2}$. The polygraphy was manually analysed according to the following definitions: Hypopnoea was defined as $\geq 30 \%$ flow reduction $\geq 10 \mathrm{~s}$ with proportional thoracoabdominal in-phase (central) or outphase (obstructive) movements followed by $\geq 3 \%$ oxyhaemoglobin desaturation. Apnoea was defined as $\geq 90 \%$ flow reduction $\geq 10$ s with proportional thoracoabdominal in-phase (central) or out-phase (obstructive) movements followed by $\geq 3 \%$ oxyhaemoglobin desaturation.

In this study, we evaluated total apnoea-hypopnea index (AHI) defined as number of respective events per hour of sleep, number of hypopnoeas per hour of sleep, number of apnoeas per hour of sleep, baseline $\mathrm{SpO}_{2}$, mean nocturnal $\mathrm{SpO}_{2}$, the percentage of recording time $<90 \% \mathrm{SpO}_{2}$ and the minimal nocturnal $\mathrm{SpO}_{2}$ recorded during sleep (nadir $\mathrm{SpO}_{2}$ ), as indices of nocturnal hypoxaemia. Moreover, during the polygraphy, one nocturnal capillary blood gas analysis (BGA) was undertaken $\left(\operatorname{artSpO} \mathrm{O}_{2}\right.$, arterial oxygen tension $\left(\mathrm{PaO}_{2}\right)$ and arterial carbon dioxide tension $\left(\mathrm{PaCO}_{2}\right)$ ).

Nocturnal hypoventilation was defined when one of the following findings in polygraphy or arterial BGA during sleep was present: (1) $\mathrm{SpO}_{2}$ during sleep $<90 \%$ for $>5 \mathrm{~min}$ with a minimum value at least $85 \%$, (2) $\mathrm{SpO}_{2}$ of $<90 \%$ for $>30 \%$ of total sleep duration or (3) $\mathrm{PaCO}_{2}$ of $>45 \mathrm{~mm} \mathrm{Hg}$ during sleep or disproportionally increased $\mathrm{PaCO}_{2}$ levels compared with wakefulness.

Patients were excluded from analysis if they had a history of symptomatic pulmonary disease unrelated to ALS or any sleep-related disease. The study was approved by the local ethics committee (Ethik-Kommission der Friedrich-Schiller-Universität Jena an der Medizinischen Fakulät. Number: 3633-12/12).

\section{Statistical analysis}

Analysis was undertaken using PASW statistics 18. Explorative data analysis and student $t$ test were used to assess group differences between (1) patients with and without dyspnoea, (2) patients with and without nocturnal hypoventilation, (3) patients with high (48-40), middle $(39-30)$ and low $(<30)$ scores on the revised ALS Functional Rating Scale (ALSFRS-R), (4) patients with bulbar and limb onset and (5) patients with a disease duration $<12$ months, between 12 and 24 months and $>24$ months. The Pearson rank correlation coefficient was used to study the relationships between clinical data, spirometry and polygraphy. Patient characteristics were expressed as mean \pm SD. A $\mathrm{p}<0.05$ was considered significant.

\section{RESULTS \\ Descriptive statistics}

In the 131 ALS patient cohort, the mean ALSFRS-R, which tracks disease severity, was $32 \pm 8$ of 48 points, and the mean disease duration was $20.5 \pm 12.8$ months from the first symptom (muscular weakness or bulbar impairment). The cohort included 46 bulbar and 85 limb onsets, which did not significantly differ in terms of disease severity (total ALSFRS-R), the respiratory subscore of the ALSFRS-R, disease duration and body mass index (table 1). However, the limb-onset group was significantly younger than the bulbar-onset group ( $t$ test, $p=0.009$ ). Reports about dyspnoea (ALSFRS-R question number 10) were made in 56\% (74 patients in total, including 30 bulbar and $44 \mathrm{limb}$ onset) and sleep-related disturbances (ALSFRS-R question 
number 11) were reported in 32\% (42 patients in total, including 21 bulbar and 21 limb onset) of the patients.

\section{Relationship between clinical data, pulmonary function tests and polygraphy}

In the first step, we wanted to determine how clinical symptom dyspnoea is reflected in different values of spirometry and polygraphy. The group reporting with versus without dyspnoea had a significantly lower ALSFRS-R $(p<0.000)$, lower VC $(p=0.002)$, lower FVC $(p=0.008)$, lower $\operatorname{artSpO}_{2}(p=0.001)$, lower $\mathrm{PaO}_{2}(0.006)$ and higher AHI $(\mathrm{p}<0.00)$, while other values such as Pimax, baseline $\mathrm{SpO}_{2}$, the nadir of $\mathrm{SpO}_{2}$, mean $\mathrm{Sp}_{2}, \mathrm{PaCO}_{2}$ and disease duration did not significantly differ (see online supplementary table S2). The respiratory question of the ALSFRS-R (number 10), asking for the severity of dyspnoea, correlated with VC $(\mathrm{R}=0.31 ; \mathrm{p}=0.001)$, FVC $(\mathrm{R}=0.291$; $\mathrm{p}=0.002), \quad \operatorname{artSpO} \mathrm{pO}_{2}(\mathrm{R}=0.224 ; \mathrm{p}=0.015), \mathrm{PaO}_{2} \quad(\mathrm{R}=0.310 ;$ $\mathrm{p}=0.001)$ and $\mathrm{PaCO}_{2}(\mathrm{R}=-0.233 ; \mathrm{p}=0.012)$ but not with the other values. It is important to note that this also means that seven patients, who did not present with dyspnoea, had an FVC lower than $50 \%$ and that 23 patients not reporting dyspnoea had an FVC lower than $75 \%$.

In the next step, we wanted to analyse the differences in spirometry parameters depending on disease severity, which can be easily assessed by the ALSFRS-R. Patients with a higher disease burden (ALSFRS-R total score $<30$ ) had a significantly lower VC $(p<0.000)$, lower FVC $(p<0.000)$, lower Pimax $(p<0.000)$, lower $\mathrm{SpO}_{2}(\mathrm{p}=0.035)$, lower $\mathrm{PaO}_{2}(\mathrm{p}=0.006)$ and lower baseline $\mathrm{SpO}_{2} \quad(\mathrm{p}=0.015)$ than patients with an ALSFRS-R of $>40$ points. With regard to disease duration, only the VC $(p=0.041)$ was significantly different (lower) in the group with the disease duration being $>24$ months compared with a disease duration of 12 months. Although it did not reach statistical significance, it is remarkable that the AHI was higher in the group with short disease duration than in the group with longer disease duration (see online supplementary table S2).

\section{Correlation between pulmonary function tests, polygraphy and BGA}

Finally, to evaluate the potential additional benefit of using a polygraphy in patients with ALS, we wanted to answer the question of how spirometry values correlate with polygraphy values. The VC correlated significantly with the baseline $\mathrm{SpO}_{2}$ $(\mathrm{R}=0.275 ; \mathrm{p}=0.005)$, the nadir of $\mathrm{SpO}_{2}(\mathrm{R}=0.208 ; \mathrm{p}=0.036)$, mean $\mathrm{SpO}_{2}(\mathrm{R}=0.293 ; \mathrm{p}=0.001)$, time under $90 \% \mathrm{SpO}_{2}(\mathrm{R}=$ -0.276; $\mathrm{p}=0.003)$ and $\mathrm{PaCO}_{2}(\mathrm{R}=-0.263 ; \mathrm{p}=0.005)$.

Nocturnal hypoventilation was observed in 67\% (32 bulbar onset, 56 limb onset, total $n=88$ ) of the ALS patients independent of their ALSFRS-R. The patient group with nocturnal hypoventilation was characterised by a significantly lower VC $(p=0.018)$, lower FVC $(p=0.019)$ and lower Pimax $(p=0.008)$ compared with the group without nocturnal hypoventilation (see online supplementary table S2). Accordingly, the prevalence of nocturnal hypoventilation correlated with VC $(\mathrm{R}=-0.217$; $\mathrm{p}=0.018)$, FVC $(\mathrm{R}=-0.219 ; \mathrm{p}=0.019)$ and Pimax $(\mathrm{R}=-0.258$; $\mathrm{p}=0.008)$. However, also in the absence of nocturnal hypoventilation, eight patients had VC of $<50 \%$ as predicted. In contrast, in 60 patients with nocturnal hypoventilation, the VC was $>50 \%$ as predicted. The detailed correlation coefficients are shown in online supplementary table S3.

\section{Follow-up}

Although the study was not designed as a prospective analysis of end points such as survival or quality of life, one could ask: what happened to these patients? Nineteen $(14 \%)$ patients of the whole cohort consisting of 131 patients did not receive NPVV due to several reasons and died after a mean of 10 $( \pm 9.7)$ months. Sixty-five $(50 \%)$ of the whole cohort received NPVV after a mean of 3 months $( \pm 6.6)$. From these 65 patients, 9 died within the following 12 months. In 8 patients from these 65 patients, the NPVV was initiated solely due to the presence of nocturnal hypoventilation (no dyspnoea, FVC $>50 \%$ of predicted). In this group, within 1 year after initiation of NPVV, no patient died. In the group where NPVV was initiated because of low FVC $(<50 \%$ of predicted) or the presence of dyspnoea, nine patients died within 1 year after initiation of NPVV. Both groups (nocturnal hypoventilation only vs low FVC or dyspnoea) did not significantly differ in terms of age, disease duration or ALSFRS-R.

\section{DISCUSSION}

To the best of our knowledge, this is the first study to provide comprehensive data on polygraphy and its correlation with other respiratory values and clinical data in a large cohort of patients with ALS. This is an important issue as respiratory function has a high impact on survival and quality of life in people with ALS. Three main aspects should be discussed in more detail.

First, the relevance of reporting dyspnoea or, maybe even more challenging, the absence of dyspnoea. The ALSFRS-R and its respiratory question (number 10) correlated with the decline of VC, FCV and changes in nocturnal capillary blood gases. Therefore, if a patient presents with dyspnoea, one can assume a relevant impairment of respiratory function and the necessity of NPPV. However, the absence of dyspnoea does not rule out a relevant respiratory impairment, as $30 \%$ of the patients with ALS not reporting dyspnoea had an FVC of $<75 \%$ as predicted. This is of special importance because a FVC of $75 \%$ rather than $50 \%$ is discussed as the threshold for the initiation of NPPV, where NPPV has a positive impact on the quality of life and survival. $^{10} 11$

Second, nocturnal hypoventilation. Our study provides additional data for the role of sleep-related breathing disorders in ALS. The loss of central ventilatory drive seems to be an important factor leading to hypopnoea and nocturnal desaturation and sleep disturbances appear at an early stage of the disease. ${ }^{12} \mathrm{We}$ observed nocturnal hypoventilation in $67 \%$ of patients with ALS independent of their ALSFRS-R or their onset type. Our patients with nocturnal hypoventilation showed significantly worse spirometry and blood gas values than patients without nocturnal hypoventilation. However, the absence of nocturnal hypoventilation does not necessarily indicate normal respiratory function, because FVC ranged in this group from 25\% to $123 \%$ as predicted. Consistent with former studies, we did not find a significant association between bulbar dysfunction and severity of respiratory events (eg, $\mathrm{AHI})^{13}{ }^{14}$ and found that sleep-related respiratory events seem to be more common in the early stage of the disease with a progressive decline in the number of events (AHI, hypopnoea and apnoea) during the disease course (although this did not reach statistical significance). It was suggested that this reflects increasing inspiratory muscle weakness. ${ }^{15}$ With increasing diaphragmatic involvement, the ability of the participant to generate an inspiratory pressure above the closing pressure of the upper airway would be reduced, resulting in fewer events being recorded. ${ }^{13} 16$

Third, awake spirometry, oximetry and nocturnal BGA. How can awake measures of $\mathrm{SpO}_{2}$ (oximetry), spirometry and nocturnal BGA be used to predict nocturnal hypoventilation? We 
found a moderate correlation between daytime/baseline $\mathrm{SpO}_{2}$ and parameters of nocturnal hypoventilation. Usually a nocturnal desaturation is unlikely where daytime $\mathrm{SpO}_{2}$ was $>94 \%{ }^{16}$ However, in our ALS cohort, 35\% (17) of the patients with a baseline $\mathrm{SpO}_{2}>94 \%$ (48) had a nocturnal desaturation where $\mathrm{SpO}_{2}$ fell $<85 \%$. This means that every third patient with ALS with a normal daytime $\mathrm{SpO}_{2}$ has nocturnal hypoventilation and is a candidate for NPPV. In several neuromuscular disorders, such as Duchenne muscular dystrophy or limb girdle muscular dystrophy, spirometry on the basis of a VC of $<40 \%$ can predict nocturnal hypoventilation. ${ }^{16}$ Moreover, in our study, the VC and FVC showed a correlation with nocturnal hypoventilation. Because nocturnal capillary blood samples are easily accessible, they are an alternative to arterial blood samples. In our study, the capillary $\mathrm{SpO}_{2}$ and $\mathrm{PaO}_{2}$ correlated with the presence of nocturnal hypoventilation.

In summary, the occurrence of chronic dyspnoea clearly indicates respiratory failure. In addition, if FVC is $<50 \%$ (or probable $75 \%$ ) as predicted, it is understood that NPPV should be initiated in patients with ALS. The question remains of how to deal with patients who do not report dyspnoea and have an FVC of $>75 \%$. Our data show that in this patient group $(n=29)$, a nocturnal hypoventilation was observed in nearly every second patient $(n=14)$. Therefore, for the question of whether NPPV should be initiated, a polygraphy does not provide useful additional information if the FVC is already $<75 \%$ as predicted. However, in patients with more or less normal lung function parameters or where lung spirometry cannot be performed adequately (eg, bulbar ALS), polygraphy can provide adequate evidence for the need of NPPV. Therefore, we propose the following algorithm regarding NPPV initiation in patients with ALS (figure 1).

In general, we believe that an early initiation of NPVV, for example, when FVC is $>50 \%$ in ALS is not detrimental. In our experience, the compliance and acceptance of NPVV in patients with ALS is better when NPVV is initiated before severe respiratory failure occurs. If a patient does not report dyspnoea and

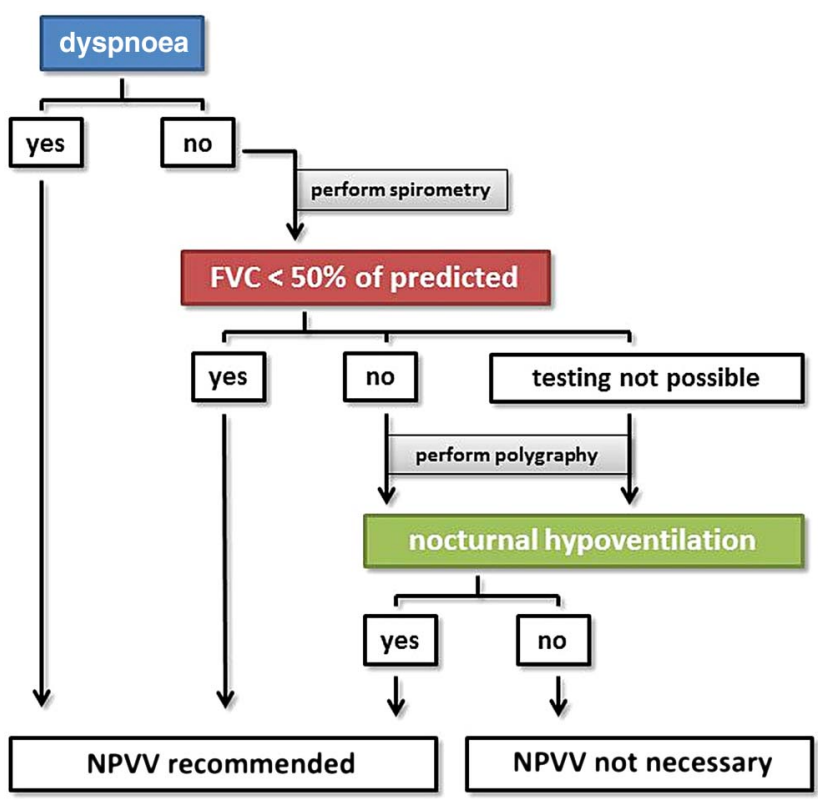

Figure 1 Proposed algorithm for the evaluation of the respiratory state and the need for NPPV (FVC, forced vital capacity; NPPV, non-invasive positive-pressure ventilation). has normal FVC and borderline evidence of nocturnal hypoventilation, we usually repeat the respiratory assessments after 3 or 6 months, depending on the progression rate. In accordance with the international trend of increased use of NPPV as the treatment of choice for respiratory disturbances in ALS every second patient was non-invasive ventilated in our cohort. It seems that overall survival after initiation of NPPV is similar to that observed in other studies. ${ }^{17}{ }^{18}$ From our data, it seems that there is a trend towards a better survival for beginning NPVV when only nocturnal hypoventilation is present. However, this was just a retrospective analysis of 1 year survival in only a few patients. There are several limitations of our study. First, this was a retrospective study of respiratory data which were assessed when the diagnosis of ALS was made in our hospital. Therefore, due to the aim of the current analysis, we did not undertake a prospective study which could provide longitudinal data concerning NPVV or survival. We did a retrospective analysis of several assessments in order to detect the ALS subgroup where polygraphy can provide us additional data concerning respiratory failure. The current study showed that polygraphy is not useful in every ALS case and also in terms of its costs it is not necessary to routinely screen every patient with this tool. To study the impact and effectiveness of polygraphy to detect early respiratory failure in the mentioned subgroup of patients, the next step should be a prospective study using the proposed algorithm with the primary end points survival and quality of life. Moreover, the additional use of other markers of early respiratory failure, such as the diaphragm needle EMG would be useful. Also transcutaneous carbon dioxide monitoring as a noninvasive measuring of arterial carbon dioxide levels would be a useful tool instead of the an arterialised ear lobe capillary blood sample. $^{19}$

Contributors TP was involved in conception and design of the work, and drafting the work. TP, TMR, KW, PG, AG, BS and JG were involved in acquisition, analysis or interpretation of data. OWW and JG were involved in revising the work critically for important intellectual content.

Funding This project is supported by the german Bundesministerium für Bildung und Forschung (BMBF) grant SOPHIA to JG under the aegis of the EU Joint Programme-Neurodegenerative Disease Research (JPND—http://www.jpnd.eu) and a BMBF grant PYRAMID to JG in the framework of the ERANET E-RARE programme (http://www.e-rare.eu). This study was undertaken in cooperation with the BMBF funded MND-NET.

Competing interests None declared.

Ethics approval Ethik-Kommission der Friedrich-Schiller-Universität Jena an der Medizinischen Fakulät (number: 3633-12/12).

Provenance and peer review Not commissioned; externally peer reviewed.

Open Access This is an Open Access article distributed in accordance with the Creative Commons Attribution Non Commercial (CC BY-NC 4.0) license, which permits others to distribute, remix, adapt, build upon this work non-commercially, and license their derivative works on different terms, provided the original work is properly cited and the use is non-commercial. See: http://creativecommons.org/ licenses/by-nc/4.0/

\section{REFERENCES}

1 Ambrosino N, Carpenè N, Gherardi M. Chronic respiratory care for neuromuscular diseases in adults. Eur Respir J 2009;34:444-51.

2 Pinto S, Carvalho Md. Breathing new life into treatment advances for respiratory failure in amyotrophic lateral sclerosis patients. Neurodegener Dis Manag 2014;4:83-102.

3 Bourke SC, Tomlinson M, Williams TL, et al. Effects of non-invasive ventilation on survival and quality of life in patients with amyotrophic lateral sclerosis: a randomised controlled trial. Lancet Neurol 2006;5:140-7.

4 Radunovic A, Annane D, Rafiq MK, et al. Mechanical ventilation for amyotrophic lateral sclerosis/motor neuron disease. Cochrane Database Syst Rev 2013;3: CD004427.

5 Lechtzin N, Scott Y, Busse AM, et al. Early use of non-invasive ventilation prolongs survival in subjects with ALS. Amyotroph Lateral Scler 2007;8:185-8. 
6 Vrijsen B, Testelmans D, Belge C, et al. Non-invasive ventilation in amyotrophic lateral sclerosis. Amyotroph Lateral Scler Frontotemporal Degener 2013;14:85-95.

7 Finder JD, Birnkrant D, Carl J, et al. Respiratory care of the patient with Duchenne muscular dystrophy: ATS consensus statement. Am J Respir Crit Care Med 2004;170:456-65.

8 Stewart $\mathrm{H}$, Eisen A, Road J, et al. Electromyography of respiratory muscles in amyotrophic lateral sclerosis. J Neurol Sci 2001;191:67-73.

9 de Carvalho M, Pinto S, Swash M. Association of paraspinal and diaphragm denervation in ALS. Amyotroph Lateral Scler 2010;11:63-6.

10 Carratù $P$, Spicuzza L, Cassano A, et al. Early treatment with noninvasive positive pressure ventilation prolongs survival in amyotrophic lateral sclerosis patients with nocturnal respiratory insufficiency. Orphanet J Rare Dis 2009;4:10.

11 Czaplinski A, Yen AA, Appel SH. Forced vital capacity (FVC) as an indicator of survival and disease progression in an ALS clinic population. I Neurol Neurosurg Psychiatry 2006;77:390-2.

12 Kimura K, Tachibana N, Kimura J, et al. Sleep-disordered breathing at an early stage of amyotrophic lateral sclerosis. J Neurol Sci 1999;164:37-43.
13 Ferguson KA, Strong MJ, Ahmad D, et al. Sleep-disordered breathing in amyotrophic lateral sclerosis. Chest 1996;110:664-9.

14 Gay PC, Westbrook PR, Daube JR, et al. Effects of alterations in pulmonary function and sleep variables on survival in patients with amyotrophic lateral sclerosis. Mayo Clin Proc 1991;66:686-94.

15 Santos C, Braghiroli A, Mazzini L, et al. Sleep-related breathing disorders in amyotrophic lateral sclerosis. Monaldi Arch Chest Dis 2003;59:160-5.

16 Piper AJ. Nocturnal hypoventilation-identifying \& treating syndromes. Indian J Med Res. 2010;131:350-65.

17 Chiò A, Calvo A, Moglia C, et al., null PARALS. Non-invasive ventilation in amyotrophic lateral sclerosis: a 10 year population based study. J Neurol Neurosurg Psychiatry 2012;83:377-81.

18 Ritsma BR, Berger MJ, Charland DA, et al. NIPPV: prevalence, approach and barriers to use at Canadian ALS centres. Can J Neurol Sci 2010;37:54-60.

19 Rafiq MK, Bradburn M, Proctor AR, et al. Using transcutaneous carbon dioxide monitor (TOSCA 500) to detect respiratory failure in patients with amyotrophic lateral sclerosis: a validation study. Amyotroph Lateral Scler 2012;13:528-32. 